\title{
Naturopathic Medicine is a Viable Therapy in the Prevention and Treatment of $\mathrm{H}_{1} \mathrm{~N}_{1}$
}

\section{By Jacob Scheer}

Vaccination and anti-virals are the current standard of care in the prevention and treatment of H1N1 influenza virus. Concerns have surfaced as to their safety, efficacy, and necessity. Evidence and ongoing studies indicate, however, that Naturopathic therapeutics, which includes Homeopathy and Herbal medicine, may offer a safe and effective adjunct or alternative to vaccination and anti-virals.

Immunization is widely thought to provide the most effective tool against a pandemic virus. The occurrence of a rare but serious adverse event, Guillain-Barre syndrome is associated with influenza immunization. It was first documented following the 1976 program against the swine influenza in the United States and is important to address in the context of the recent spread of the novel influenza A (H1N1) in humans. Minimizing the risk of severe adverse events is an important goal when developing immunization policies for interpandemic use of novel influenza vaccines and implementing mass immunization programs ${ }^{2}$.

On September 28, 2009, the Globe and Mail, reported a "perplexing" Canadian study linking H1N1 outbreaks to seasonal flu shots which has thrown the national influenza plans into disarray and is testing public faith in the government agencies responsible for protecting the nation's health. Distributed for peer review, the study confounded infectious disease experts in suggesting that people vaccinated against seasonal flu are twice as likely to catch swine flu.

According to the World Health Organization, alternative medicine has been the standard of care for billions of people world wide. There are a host of herbs that enhance and support the function of the immune system. Herbs such as Boneset and Vervain have been used traditionally in the treatment of influenza and influenza like symptoms ${ }^{1}$. Immune enhancing herbs such as Echinacea, Andrographis and Picrorrhiza support the body's defence against the virus. Additionally,

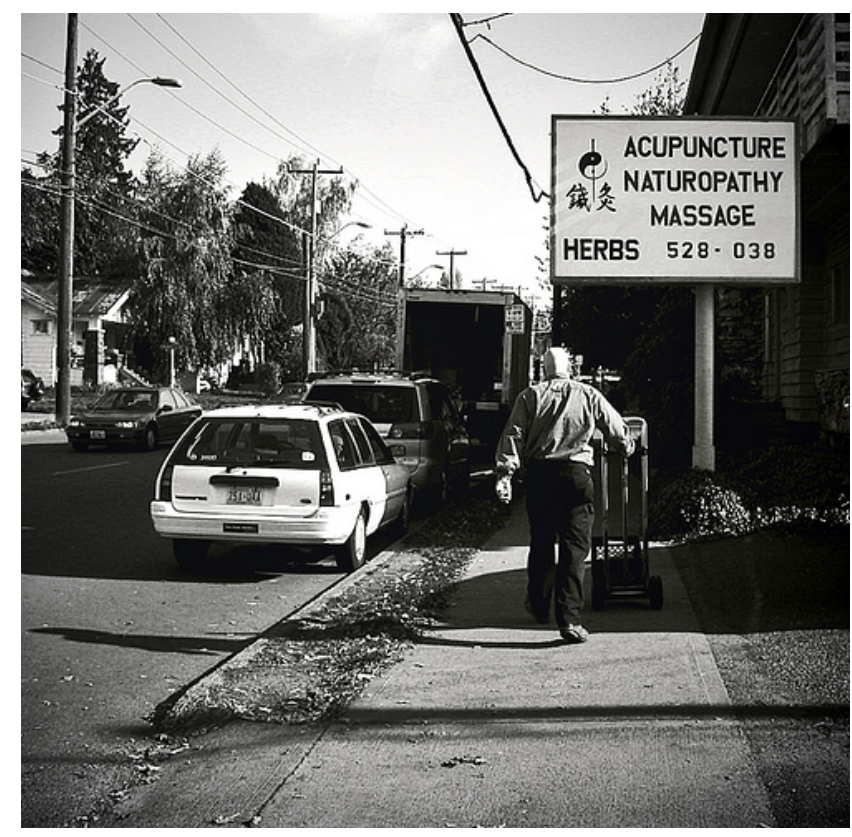

studies have shown that many herbs exhibit antiviral, anti-bacterial and anthelmintic properties ${ }^{6}$. Finally, the Physician's Desk Reference (PDR) for Herbal Medicines lists the following herbs for the treatment and symptomatic relief of influenza: Buttercup; Cinnamon; Colt's Foot; Dog Rose; Chamomile; Eucalyptus; Elder; Horseradish; Oats; Quinine; and Wild Indigo ${ }^{7}$.

Homeopathy, a highly systematic method of medical therapeutics and clinical evaluation, has been effective in the treatment of influenza and influenza-like symptoms for hundreds of years. The medicines used in this form of alternative medicine are chosen according to the Law of Similars (the concept of like curing like), a fundamental Homeopathic principle. It is based upon the observed relationship between a medicine's ability to produce a specific constellation of signs and symptoms in a healthy individual and the same medicine's ability to cure a sick patient with similar signs and symptoms ${ }^{8}$. References to the Homeopathic treatment of influenza or grip date 
back to the 1880 's ${ }^{3}$. Dr. James Kent in his book "Repertory of the Homeopathic Materia Medica" first published in 1881 refers to the treatment of influenza in the rubric: extremities, pain, influenza, etc. The Homeopathic remedies in this rubric that were used to successfully treat influenza include; aconite, bryonia, euphrasia, eupatorium perforliatum, and gelsemium. These medicines are still used successfully throughout the world to treat influenza and influenza-like symptoms.

In 1989, the following controlled clinical trial was conducted to assess the effectiveness of a Homoeopathic preparation in the treatment of influenza-like syndromes. The Homeopathic drug was Oscillococcinum, manufactured by Boiron Laboratories, made from Anas Barbariae Hepatis and Cordis Extractum HPUS 200 C. In the trial, 237 volunteers received the test drug and 241 were assigned to placebo. The A HINl influenza virus was isolated in the study region 7 days after the study managers issued the instruction to start including patients in the experiment. $17.1 \%$ of the participants from the active drug group recovered within 48 hours of treatment compared to $10.3 \%$ from the placebo group ( $p$ value 0.03 ). Volunteers with an influenza-like syndrome who received the homoeopathic preparation showed a greater early recovery rate, within 48 hours of treatment, than those who received placebo ${ }^{4}$. To date it is the only known clinical trial for H1N1. I would recommend further studies to substantiate the benefits of Naturopathic therapeutics in the the treatment of H1N1.

Is Naturopathic or alternative medicine a viable therapy in the prevention and treatment of H1N1? My professional expertise and anecdotal evidence would cry out, "Absolutely!" I am, however, able to constrain emotional exuberance knowing that the experiences and results of the past along with the current clinical evidence support my professional conviction.

\section{Acknowledgements}

I would like to thank Jerome Singh (Join Centre of Bioethics, University of Toronto), Dr. Dena Bain Taylor (Director, Health Sciences Writing Centre, University of Toronto), and Dr. Mark Bernstein (Professor of Surgery, University of Toronto) for their assistance in preparing this manuscript.

\section{References}

1. Barnes J, Anderson LA, Phillipson JD, Herbal Medicines A guide for healthcare professionals, 2nd edition. Pharmaceutical Press. London. 2002

2. Evans D, Cauchemez S, Hayden FG, "Prepandemic" Immunization for Novel Influenza Viruses, "Swine Flu" Vaccine, Guillain-Barré Syndrome, and the Detection of Rare Severe Adverse Events, The Journal of Infectious Diseases 2009;200:321-328

3. Farrington H, A Clinical Materia Medica, Indian Books and Periodicals Syndicate, New Delhi, 1908

4. Ferley JP, Zmirou D, D'Adhemar D, Balducci F, A controlled evaluation of a homoeopathic preparation in the treatment of influenza-like syndromes, Br. J. clin. Pharmac. (1989), 27, 329-335

5. Kent JT, Repertory of the Homeopathic Materia Medica, B. Jain Publishers Pvt. Ltd 1986

6. Mills S, Bone K, Principles and Practice of Phytotherapy Modern Herbal Medicine, Churchill Livingstone, 2000

7. PDR for Herbal Medicines Second Edition, Thomson, 2000

8. Pizzorno JE, Murray MT, A Textbook of Natural Medicine, John Bastyr College Publications, Seattle, Washington 1985

9. World Health Organization (WHO) Global Alert and Response

www.who.int/csr/disease/swineflu/en/index.html

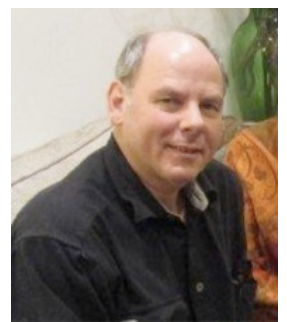

Author Profile

Jacob Scheer recently received a MHSc degree in Bioethics at the University of Toronto. Although his main research interests are in the area of clinical ethics, he also holds degrees from the Canadian Memorial Chiropractic College (Doctor of Chiropractic) and the Canadian College of Naturopathic Medicine (Naturopathic Doctor). 